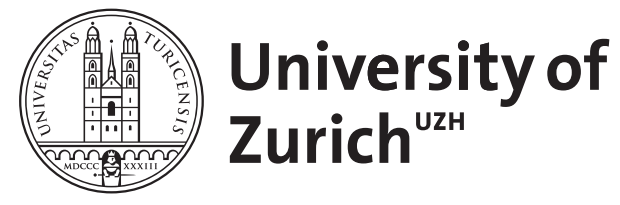
Archive

University of Zurich

University Library

Strickhofstrasse 39

CH-8057 Zurich

www.zora.uzh.ch

Year: 2009

Introduction: Cognitive issues in geographic information visualization

Fabrikant, Sara I ; Lobben, A

DOI: https://doi.org/10.3138/carto.44.3.139

Posted at the Zurich Open Repository and Archive, University of Zurich

ZORA URL: https://doi.org/10.5167/uzh-27610

Journal Article

Originally published at:

Fabrikant, Sara I; Lobben, A (2009). Introduction: Cognitive issues in geographic information visualization. Cartographica, 44(3):139-143.

DOI: https://doi.org/10.3138/carto.44.3.139 


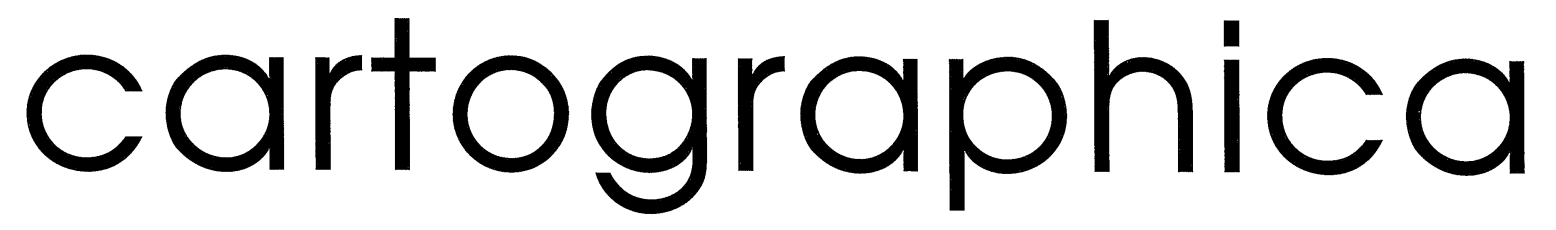

the international journal for geographic information and geovisualization 


\section{Cartographica / Contents}

\section{Volume 44, Number 3, Fall 2009}

\section{Special Issue: Cognitive Issues in Geographic Information Visualization}

Guest Editors' Introduction

Sara Irina Fabrikant and Amy Lobben

Applications of Signal Detection Theory to Geographic Information Science

Amy L. Griffin and Scott Bell

fMRI and Human Subjects Research in Cartography

Amy Lobben, Megan Lawrence, and Judy M. Olson

Naïve Cartography: How Intuitions about Display Configuration Can Hurt Performance

Mary Hegarty, Harvey S. Smallman, Andrew T. Stull, and Matt S. Canham

Navigation Tasks with Small-Display Maps: The Sum of the Parts Does Not Equal the Whole Julie A. Dillemuth

Issues of Change Detection in Animated Choropleth Maps

Kirk Goldsberry and Sarah Battersby

Colour-Enhanced Star Plot Glyphs: Can Salient Shape Characteristics Be Overcome?

Alexander Klippel, Frank Hardisty, Rui Li, and Chris Weaver

Advice to Contributors

The Canadian Cartographic Association / L'Association cartographique canadienne

The International Cartographic Association / L'Association cartographique internationale 


\title{
Introduction: Cognitive Issues in Geographic Information Visualization
}

\author{
Sara Irina Fabrikant \\ Department of Geography / University of Zurich / Zurich / Switzerland \\ Amy Lobben \\ Department of Geography / University of Oregon / Eugene / OR / USA
}

In recent years geovisualization has morphed into the field of "geovisual analytics," emphasizing human exploration through highly interactive visual interfaces to geographic information, taking advantage of perceptual and cognitive abilities to recognize and process patterns and outliers from visual displays, linking these patterns and outliers to existing mental schemata and knowledge bases, and eventually arriving at an appropriate spatially relevant course of action or decision given the visual input (Chen and others 2008). Visual analytics is based on the intuition that highly interactive and dynamic depictions of complex and multivariate databases amplify human capabilities for inference and decision making, as they facilitate cognitive tasks such as pattern recognition, imagination, association, and analytical reasoning (Thomas and Cook 2005). One may wonder, however, why success stories in this vibrant (albeit still young) research field seem practically non-existent (Johnson and others 2006). By "success story" we mean a scenario in which the creators of a visual analytics tool or approach are not the sole user group, or significant insights into real-world data were generated for decision makers, or target users could actually use the tool to solve real research problems or perform the day-to-day tasks at hand.

Unsurprisingly, one pervasive theme underlying many current (geo-)visualization research challenges is the difficulty of effectively evaluating highly interactive visualization tools and complex displays and identifying their potentially positive influence on exploratory data analysis, knowledge extraction, and learning. While research within the visual analytics and geovisual analytics communities has focused predominantly on building tools and the highly interactive computer-human interfaces that accompany them, fundamental investigations based on empirical evidence of how, when, and why human inference, analytical reasoning, and decision making with visual displays work have received considerably less attention. We still know little about the effectiveness of graphical displays for exploratory data analysis, problem solving, knowledge exploration, learning, and decision making. This lack of understanding is particularly noteworthy because the predecessor of the current International Cartographic Association (ICA) Commission on Geovisualization (then called the ICA Commission on Visualization and Virtual Environments) had already identified this problem at the beginning of the twenty-first century as one of the key challenges for geovisualization research - namely, the necessity to focus on cognitive issues and usability (MacEachren and Kraak 2001). Specifically, these authors point to the need to develop a theoretical framework, based on cognitive principles, to support a humancentred approach to geovisualization. In a 2006 report on visualization research challenges that not only summarizes visualization achievements but also discusses major obstacles blocking the discipline's advancement, Johnson and others (2006, 9-10) emphasize that if we are to accelerate progress in visualization and visual communication, the investigation of the nature, options, limitations, and effects of human perception, human cognition, and the visual exploration experience needs 
to be addressed more systematically. Along these lines, Gennady Andrienko and others (2008) point out that visual analytics methods developed must be cognitively adequate for successful inference and that tools must be usable for analysis and decision making. One appropriate starting point for constructing effective and efficient visual analytics tools is to frame developments within context of cognitive theories, long-standing empirically evaluated design principles, and related empirical studies on visual displays.

This challenge inspired the idea for this special issue of Cartographica. The aim of the issue is to showcase current fundamental empirical research and state-of-the-art evaluation methods within the interdisciplinary empirical research domain of geographic information visualization and cognition, as well as to further stimulate new ideas for future empirical work. The papers originated from contributions presented in two paper sessions at the Association of American Geographers (AAG) annual meeting in Boston, Massachusetts, on 18 April 2008. These sessions, ${ }^{1}$ sponsored by the Cartography, GIS, and Behavioral Geography and Environmental Perception Specialty Groups of the AAG, were the result of an internationally disseminated open call for papers initiated by the guest editors of this issue. The call for papers specifically aimed to attract theoretical and empirical contributions exploring the human-geovisualization display interface. Topics of interest listed in the call for papers included

- geovisualization design research (2- and 3D, static, animated and interactive, virtual and immersive, mobile, etc.)

- the application of cognitive theories and methods to understanding visuo-spatial displays and tool use for inference and decision making

- the application of visuo-spatial displays and tools to understanding spatial cognition

- spatial reasoning, inference, and decision making with visuo-spatial displays and tools

- human-visualization interaction research

The high level of interest in cognitive work raised by the call for papers ultimately resulted in the participation of nine presenters based at universities all over the United States, as well as one from Switzerland, and attracted an audience of more than 50 people for each session. The enthusiastic response to the AAG sessions, from presenters and the audience alike, and the appearance of this special issue suggest that the interdisciplinary topic of cognition in geovisualization is not only timely and relevant for the GIScience, geovisualization, and cartography research communities but also of significant interest to a broader interdisciplinary audience, including spatial cognition researchers. Geovisualization research seems alive and well, considering the growing numbers of workshops, meetings, and conferences, for example, organized by the ICA Commission on GeoVisualisation ${ }^{2}$ or its active members and the resulting papers in proceedings, journals, and special journal issues in GIScience, cartography, and other cognate fields.

After the AAG conference, the session organizers invited presenters to submit full papers for review and potential publication in Cartographica; this invitation was followed by a second open call for papers, disseminated digitally through various e-mail lists around the globe. The seven full papers received included four submissions extended from oral AAG presentations and three external submissions responding to the second call for papers. Each submission was double-blind reviewed by two to three external reviewers as well as by the journal editor and the guest editors. The journal editor handled the co-authored contribution by one of the guest editors separately. The six papers that appear in this special issue are the result of this multi-stage review process.

While the original topics of interest proposed in the calls for papers included more specific foci, we collapsed the topics into two conference sessions titled "Methods" and "Domains," while recognizing that some papers fit neatly into both sessions. This special issue features articles that are unique in offering both novel methods for empirical work in geovisualization and new domains for applying these methods. Because a rigid categorical breakdown does a disservice to these materials, we will not presume to pigeonhole each article into one of the specific foci mentioned above. In these articles, new methods for the geovis community are presented in domain contexts (Griffin and Bell; Lobben, Lawrence, and Olson); new domains are presented with established methods from other research disciplines (Hegarty and others; Dillemuth); and hybrid papers address current cartographic constructs in new ways and apply methods from other research areas to answer fundamental cartographic questions (Goldsberry and Battersby; Klippel and others).

Two studies propose knowledge transfer from established evaluation methods in psychology to solve fundamental behavioural research questions in geography using visual displays. Amy L. Griffin and Scott Bell suggest the application of signal detection theory (SDT), while Amy Lobben and others propose neuroimaging, and specifically functional magnetic resonance imagine (fMRI), to further expand the cartographic research methodology. Griffin and Bell suggest that SDT offers cartography, GIScience, and visualization an analytical method with the potential to enhance unique properties associated with much past and current (and, likely, future) research. They suggest that SDT is potentially applicable in research whose objectives include decision making involving multiple choices, as, they argue, does much of our research. Griffin and Bell characterize SDT as allowing GIScience researchers to posit questions that lead to four potential 
responses: a hit (correctly identifying an object), a false alarm (identifying a false object), a miss (failing to identify the correct object), and correct rejection (correctly rejecting the incorrect object). In arguing for the usefulness and effects of SDT, they use the example of a human navigation study in which volunteers were asked to follow a route depicted on one of three navigation aids: a survey map, a strip map, and a segmented strip map. Half the participants studied the map prior to navigation and traced the route from memory, while half traced the route while carrying the map for reference. The authors' application of SDT allowed them to discover an effect of navigating from memory versus navigating with a map: participants who travelled with the map were less likely to remember landmarks. Griffin and Bell suggest that such a discovery would likely not have been possible without the application of SDT. In addition to the case study, they suggest several potential applications of SDT in geography and provide general examples. They further suggest that SDT has the potential to be useful in research that includes non-human (i.e., machine) decision making.

The paper by Lobben and others is the first fMRI review study to appear in the peer-reviewed GIScience literature. Their study provides a potential foundation for the inclusion of neuroimaging methods - specifically fMRI - in cartographic research, situating their argument within well-known research areas in cartographic design: visual search, perceptual grouping, and figure-ground. Lobben and others review studies from the neuroscience literature that are similar (in terms of terminology and concepts, if not of stimuli) to these cartographic design constructs. The objective of the paper is not to suggest activation associated with cartographic design (as the authors point out, such studies have yet to be conducted) but to suggest a framework for the development of fMRI-related cartographic design research.

New domains are proposed and investigated by cognitive psychologists Mary Hegarty and others and by a geographer, Julie A. Dillemuth, who approaches a classic empirical research area in behavioural geography, navigation, from a new perspective. Hegarty and others propose and investigate "naïve cartography," which they suggest refers to individuals' intuitions about visual displays and their design choices and opinions. They document an interesting dichotomy: naïve cartographers tend to prefer $3 \mathrm{D}$ over $2 \mathrm{D}$ representations and to prefer more realistic (and more complex) representations to simpler, more streamlined representations of the same construct. But principles of cartographic and graphic design suggest the opposite: abstraction, simplification, and generalization. The authors conducted a study to ascertain whether naïve cartographers distinguish between display preferences and display efficiencies. They also investigated the effect of experience on naïve cartographic intuition. In the first of three studies, they disseminated a survey to more than 700 undergraduate students (a group they define as naïve cartographers); the respondents preferred enhanced displays with animation and realism. For the second study, Hegarty and others altered their questionnaire and selected meteorologists - who regularly work with visual displays - as an "expert" group. The results revealed similar a preference for enhanced displays with animation and realism. The authors further investigated the relationship between preference and performance of map tasks among naïve undergraduates and meteorologists. Their experimental design included a spatial abilities task and tasks requiring participants both to make inferences and to rate preference between maps that varied in terms of realism. The results of this third study revealed that enhanced realism on weather maps led to declines in performance for both groups but that participants still preferred the enhanced maps. Hegarty and others' overall results suggest a dichotomy between preference and effectiveness: map users' preference for a display type is not necessarily a good indication of the effectiveness of that display type. These results are another good, empirically validated illustration of the common-sense notion that what people think they want is not always what is best for them. Hegarty and others offer a new cartographic construct, naïve cartography, along with some interesting supporting empirical data.

While previous research has been conducted on map use and navigation, Dillemuth provides a new perspective through her investigation of the effects of small digital mobile map displays (i.e., maps on cell phones or personal digital assistants) on map use, navigation, and cognitive map development. Her volunteers, grouped into one of four conditions $(10 \%, 25 \%, 40 \%$, or $100 \%$ map view), were asked to perform the same tasks: distance/direction estimates, landmark recall, and sketch mapping. All participants also completed a sense-of-direction questionnaire as well as a perspective taking/spatial orientation test. Overall, as map extent decreased (from 100\% to $10 \%)$, accuracy declined and response times rose. Though no difference in confidence was observed between group conditions, Dillemuth found that higher spatial abilities (as measured by the instruments used in the study) correlated with higher performance in the $10 \%$ viewing condition.

Finally, two papers provide an effective hybrid approach. Both Kirk Goldsberry and Sarah Battersby and Alexander Klippel and others address familiar cartographic topics: map animations and multivariate map symbols (i.e., glyphs). In both cases, however, the authors add a new wrinkle by using methods borrowed from other research fields to evaluate and understand users' perception of and understanding from visual displays. Goldsberry and Battersby focus on enhancing the understanding of animated choropleth maps; in doing so, they offer new methodologies for investigating the misunderstanding of these 
maps. Their study systematically explores why animated maps are difficult to read. In addition to a review of relevant literature in the areas of change blindness, foveal and peripheral attention, smooth and abrupt changes, visual variables, spatial attention, and magnitude of change, the authors address potential causes of misunderstanding or misreading of animated choropleth maps, including the limits of the human perceptual system and the limits of the research methodologies currently employed to understand these causes. Moreover, they provide a foundation for launching a systematic methodological approach to empirical research designed to investigate the difficulties associated with animated choropleth maps. This foundation includes methods for quantifying change in animated choropleth maps based on detecting and quantifying magnitude of change. Goldsberry and Battersby suggest that magnitude of change is a critical confounding factor in understanding animated choropleth maps, and propose two methods for quantifying magnitude of change. Basic magnitude of change (BMOC) is a count of the enumeration units in the animated choropleth map that change between the origin-state and the destination-state (or the beginning and ending of the animation); the proportion of change is then normalized by dividing the changed units by total number of units. Magnitude of rank change (MORC) provides an indication of the severity of change between the origin-state and the destination-state of an enumeration unit.

Klippel and others also tackle a fundamental cartographic design issue: the influence of perception on the extraction of meaning from map symbols. Following on from their previous research, they conducted two experiments on shape symbolization, including perceptual classification, and categorization. Eight-point star-plot glyphs were used to represent data about future cars, with each variable (price, weight, safety rating, maximum speed, miles per gallon, acceleration, emissions, and interior space) represented by one of the eight rays. Because the findings of a previous experiment revealed that singlespike glyphs resulted in unique grouping properties, Klippel and others introduced colour as a variable, in an attempt to offset the influence of shape. Their study also included a control group that viewed varying glyph shapes not related to the data set (geometric shape comparisons only). Their volunteers were assigned to one of two groups. All participant groups viewed 81 glyphs; eight variables/ rays received four different treatments in the glyph designs. Four of the variables were assigned values categorized into three classes (low, medium, high); the other four were assigned randomized values within the "high" category. Participants were asked to view the glyphs and create a categorization; they were then asked to explain their grouping rationale. The empirical results, not surprisingly, reveal that adding meaning to the glyphs (in this case, values for the variables/rays) slowed decision making (measured by reaction time or time to finish) relative to shape-only classification. Like Goldsberry and Battersby, Klippel and others also introduce a new analytical method, KlipArt, designed and applied to analysis of differences in icon classification. Their discussion suggests that future research should include an evaluation of point-symbol properties and their effects on pre-attentive processing to facilitate visual search, perceptual grouping/ categorization, and decision making.

This point brings this special issue near full circle. Lobben and others also address methods to investigate perceptual grouping; what if Klippel and others applied fMRI to investigate the brain-activation differences between the four glyph conditions? Perhaps they could hypothesize the observation of different activation patterns associated with each of the four conditions.

With this collection of six papers, we hope to have raised interest in cognitive issues in geovisualization and to stimulate new research avenues toward more systematic investigations of the effects of human perception and cognition with highly interactive, multivariate, multidimensional, and dynamic geovisualization displays.

\section{Acknowledgements}

This special issue would not have been possible without the commitment, perseverance, and help of many people. We would like to thank Cartographica Editor Jeremy Crampton, as well as Co-editors Clifford Wood and Roger Wheate and Editorial Assistant Laura Wilson, who were an invaluable help in making this special issue happen. Special thanks go to the reviewers for their excellent support under considerable time constraints. Last but not least, we thank all the authors who took the time and effort to submit their best work to the 2008 AAG sessions in Boston and to this special issue.

\section{Author Information}

Sara Irina Fabrikant, Associate Professor of Geography and Head of the Geographic Information Visualization and Analysis Unit, Department of Geography, University of Zurich, Winterthurerstr. 190, CH-8057 Zurich, Switzerland. Tel: +41 (44) 635-5150. Fax: +41 (44) 635-6848. E-mail: sara@geo.uzh.ch. Professor Fabrikant's research and teaching interests lie in geographic information visualization and (geo)visual analytics, GIScience and cognition, and graphical user interface design and evaluation, including dynamic cartography:

Amy Lobben, Assistant Professor, Department of Geography, 1251 University of Oregon, Eugene, OR 97403 USA. Her research and teaching interests lie in environmental and representational perception, map use, navigation, spatial and environmental cognition, 
and geographic/cartographic applications in neuroimaging (specifically fMRI).

\section{Notes}

1. The sessions are available online at $h t t p: / / c o m m u n i$ cate.aag.org/eseries/aag_org/program/SessionDetail.cfm? Session ID =5261 (session 1) and http://communicate.aag .org/eseries/aag_org/program/SessionDetail.cfm?SessionID $=6181$ (session 2)

2. The homepage of the ICA Commission on GeoVisualization can be accessed at http://geoanalytics.net/ica/.

\section{References}

Andrienko, G., N. Andrienko, J. Dykes, S.I. Fabrikant, and M. Wachowicz. 2008. "Geovisualization of Dynamics, Movement and Change: Key Issues and Developing
Approaches in Visualization Research." Information Visualization 7/3: 173-80.

Chen, J., R. Roth, A. Naito, E. Lengerich, and A. MacEachren. 2008. "Geovisual Analytics to Enhance Spatial Scan Statistic Interpretation: An Analysis of U.S. Cervical Cancer Mortality." International Journal of Health Geographics 7: 57. Available at http://www.ij-healthgeographics.com/content/7/ $1 / 57$.

Johnson, C.R., R. Moorhead, T. Munzner, H. Pfister, P. Rheingans, and T.S. Yoo. 2006. NIH-NSF Visualization Research Challenges Report Los Alamitos, CA. IEEE Press.

MacEachren, A.M., and M.-J. Kraak. 2001. "Research Challenges in Geovisualization." Cartography and Geographic Information Science 28: 3-12.

Thomas, J.J., and K.A. Cook. 2005. Illuminating the Path: Research and Development Agenda for Visual Analytics. Richland, WA: IEEE Press. 


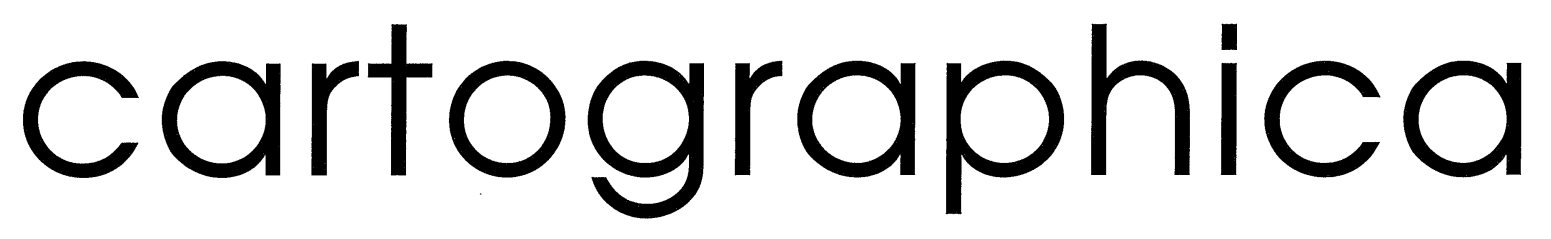

the international journal for geographic information and geovisualization 